Article

\title{
Intelligent Thermal Comfort Controlling System for Buildings Based on IoT and AI
}

\author{
Yafei Zhao *, Paolo Vincenzo Genovese and Zhixing Li \\ School of Architecture, Tianjin University, Tianjin300072, China; pavic@163.com (P.V.G.); \\ zhixinglee@hotmail.com (Z.L.) \\ * Correspondence: zhaoyafei@tju.edu.cn; Tel.: +39-3313-324-413
}

Received: 31 December 2019; Accepted: 7 February 2020; Published: 10 February 2020 updates

\begin{abstract}
With the improvement of technologies, people's demand for intelligent devices of indoor and outdoor living environments keeps increasing. However, the traditional control system only adjusts living parameters mechanically, which cannot better meet the requirements of human comfort intelligently. This article proposes a building intelligent thermal comfort control system based on the Internet of Things and intelligent artificial intelligence. Through the literature review, various algorithms and prediction methods are analyzed and compared. The system can automatically complete a series of operations through IoT hardware devices which are located at multiple locations in the building with key modules. The code is developed and debugged by Python to establish a model for energy consumption prediction with environmental factors such as temperature, humidity, radiant temperature, and air velocity on thermal comfort indicators. By using the simulation experiments, 1700 data sets are used for training. Then, the output PMV predicted values are compared with the real figure. The results show that the performance of this system is superior to traditional control on energy-saving and comfort.
\end{abstract}

Keywords: Internet of Things; thermal comfort; control system; energy consumption; artificial intelligence

\section{Introduction}

With the increasing proportion of building energy consumption, the reasonable and efficient prediction has gradually attracted the attention of the industry. Especially under the influence of the development trend of new-generation information technology, using intelligent methods to predict building energy consumption has been widely used. Cutting-edge technologies based on big data, cloud computing, machine learning, and artificial intelligence algorithms are combined with traditional architectural design theories. Building energy consumption is an interdisciplinary research direction consisting of multiple influencing factors. There is a complex correlation between various factors. As the proportion of building energy consumption is increasing, reasonable and efficient building energy prediction is gradually concerned. The comfort of the human living environment is a major concern of society after entering the information technology era. With people's increasing satisfaction to indoor life and activities, how to improve the life quality of building occupants and build a suitable living environment is a problem that is valued in the technical field. The intelligentization of indoor thermal environments has also received more and more attention. The IoT hardware plays an important role in forming a good indoor thermal and humid environment.

Building energy consumption is an interdisciplinary research direction composed of multiple influencing factors, and there is a complex correlation between each factor. Before the rapid development of artificial intelligence technology, the computing power of hardware had not yet met the needs of scientific research. The calculation model of building energy consumption cannot achieve accurate 
prediction, and the error is relatively large. In recent years, the development trends of computer science, applied mathematics, statistics, and semiconductor hardware have brought iterative progress in artificial intelligence technology. This makes the simulation, analysis, prediction.

\section{Literature Review}

Big data mainly refers to data management on a certain scale. Due to the increase of the data volume, speed, and type, traditional methods cannot be used to proceed [1]. The artificial intelligence technology represented by machine learning and deep learning algorithms has a great dependence on datasets. Only when the original data is large enough can the trained algorithm be more accurate. Therefore, before applying the model, it is necessary to obtain enough data for repeated training.

In the traditional building with related planning and landscape fields, big data was firstly applied to Geographic Information System (GIS), including a variety of spatial information such as land conditions, terrain, and climate. As for the single building, its automated development is mainly reflected in the field of intelligent building, with small but higher accuracy. The complexity of intelligent building systems is high. Meanwhile, the physical structure, indoor environment, and operating systems of the entire building are monitored and analyzed. It can develop the building in the direction of intelligence. With the help of building big data system, it can analyze the use of the building in real-time, integrate the related information of the surroundings, and let the building make optimal adjustments according to the actual situation [2]. In the process of optimal adjustment, IoT sensors and controllers are usually targeted in the building. The organic combination of physical devices and computer algorithms plays a role similar to the Internet of Things.

The sample data set comes from the real building operation data in the past, which is usually static, and the use of big data transforms it into dynamic updates. Compared to traditional statistical data sampling, the full sample data of big data has the characteristics of multi-source, open, synchronized, etc. In this real-time acquisition and optimization scenario, through analysis of energy consumption information such as building physics, ventilation, and insulation, real-time analysis and correction of building operation deficiencies can be made. And this can meet the specifications of temperature, ventilation efficiency, noise, illuminance, air quality, and meet the evaluation standards of healthy buildings.

There are many application scenarios for big data, such as dynamic analysis of large-scale public buildings or a mobile phone that is carried by a resident that can be used as a sensor to analyze the indoor population density and the emotional evaluation of the building space in real-time. Therefore, a reasonable improvement decision is proposed for subsequent building operations. There is some commonly used software for building big data: ArcGIS, Rhino, Grasshopper, and spatial syntax tool Depthmap. Professional data analysis languages like Python and R are also widely used. Generally, a variety of tools are used to solve complex problems across disciplines.

\subsection{Artificial Intelligence Algorithms For Building Energy Prediction}

\subsubsection{Acausal and Reverse Modeling}

Acausal modeling and reverse modelling are a set of opposite modeling methods used in building energy analysis. Acausal modeling completely follows the engineering principles for energy consumption prediction. This method does not need to be combined with the actual energy consumption data of the building, so it is widely used in the design phase of new buildings [3]. Reverse modeling is a data-driven method. The historical operation data of the building is obtained through a series of technical means in the early stage. Then, the data classification is used as the data set, and a certain algorithm is used to establish the analysis model and prediction model of the target building to simulate the future energy consumption of the building. Common reverse modeling methods include black box method, gray box method, and so on. 
The black box method is to simplify the research object to a black box with a specific algorithm and to input the front-end data through operations, analysis, processing, transformation, and transmission, and finally output the results. It is an effective research method. In recent years, artificial intelligence algorithms for building energy prediction are mostly black box methods or its extension techniques. This chapter will focus on several mainstream black box methods: artificial neural network and support vector machine.

\subsubsection{Artificial Neural Network (ANN)}

ANN is an information processing method similar to the structure and function of the human brain. Proposed in the 1940s, it has the characteristics of non-linear and parallel distribution structure. The algorithm is simple and accurate, so it is widely used in many fields. Many scholars have conducted studies on the prediction of building energy consumption. Neto et al. used ANN and EnergyPlus to collect data of the Administration Building of Sao Paulo University as an example to predict building energy consumption. They use parameters such as building facade and weather data to make predictions [4]. Kalogirou et al. developed an ANN-based program to simulate energy consumption according to different exterior wall materials and trained dynamic simulation data [5]. Chen et al. established an ANN energy consumption prediction model for HVAC systems in office buildings, but the model has fewer input parameters [6]. Yu et al. proposed a method based on ANN for energy consumption prediction and evaluation of indoor comfort, and optimized it through genetic algorithms [7].

Back propagation (BP) is a typical acausal neural network method, which is currently most widely used. The least mean squares (LMS algorithm) and model make the prediction result of BP neural network reliable, which makes BP very different from the earlier Madaline network algorithm. BP neural network has multi-level characteristics. Typical structures include an input layer, calculation layer, and output layer (Figure 1). The working principle is, in the process of acausal propagation, if the result of the output layer meets the conditions, it means that the training is passed, and the calculation of the next neuron can be performed. If the output result does not meet the conditions, the reverse operation is performed to return the signal to the original path. Then, it has to regenerate a result by modifying the corresponding parameters or go to the next neuron next to it. In a typical BP structure, a neuron in each layer can only affect the two layers adjacent to it and cannot execute across layers until the global optimal solution is output.

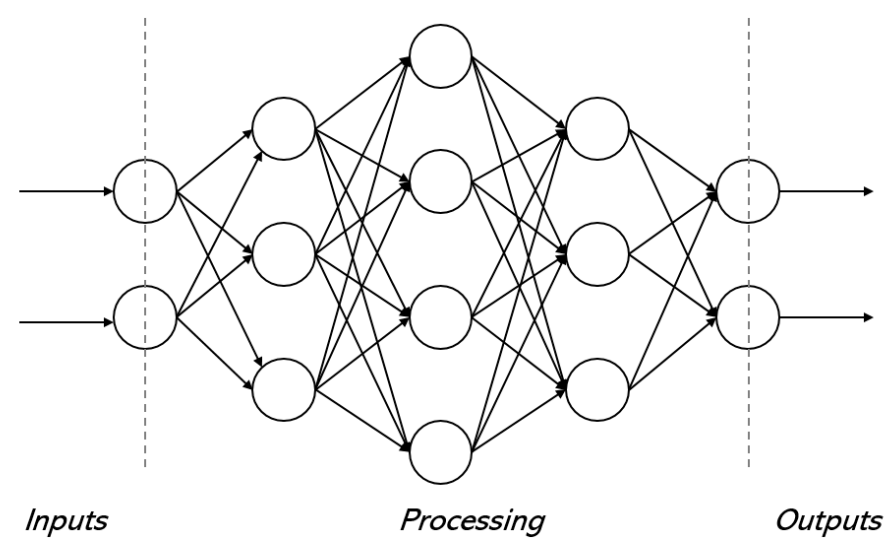

Figure 1. Typical BP Neural Network Structure.

Since the typical BP neural network structure uses offline historical data, the input results may have limitations. To address this limitation and make the results more accurate, Zhang proposed to add a dynamic compensation mechanism to the BP neural network structure [8]. This reversed mechanism can take the output result as a new condition, import it into the input layer and start a new 
round of dynamic adjustment (Figure 2). Li et al proposed improvement and correction measures for the error defect of the model [9].

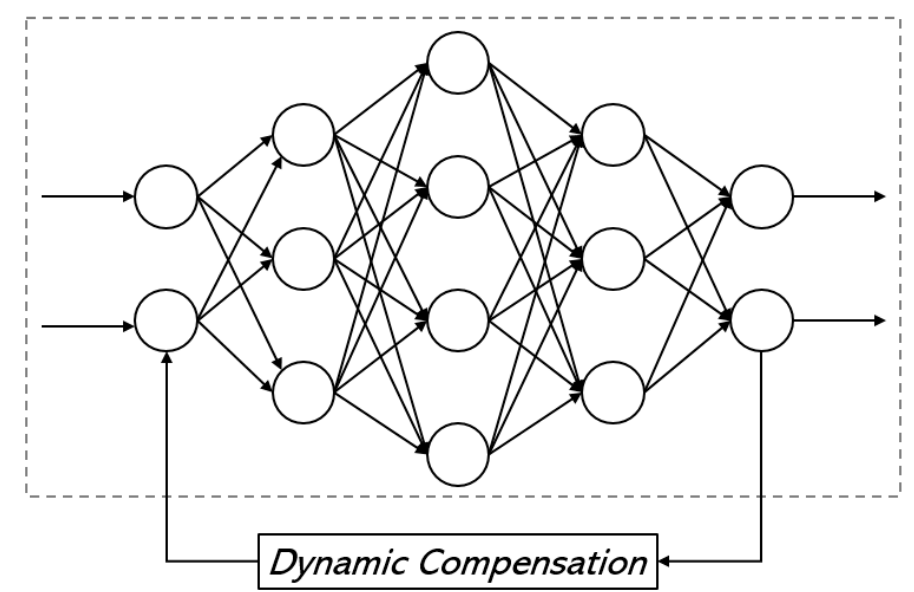

Figure 2. BP Neural Network Structure Dynamic Compensation Mechanism.

\subsubsection{Support Vector Machine (SVM)}

SVM model can effectively solve the nonlinear building energy consumption simulation problem. It is derived from statistical theory and has a strict mathematical theoretical foundation. It is a new type of machine learning algorithm that differs greatly from the bionic theory, such as ANN. Many scholars have also done a lot of research on the application of SVM to building energy consumption. Ahmad et al. summarized the comparison of power consumption prediction between SVM and ANN in detail [10]. Dong et al. used the SVM method to predict building energy consumption in tropical regions by analyzing multiple parameters [11]. Li et al. established a model for building cold load prediction based on SVM to predict the cooling load of buildings per hour [12].

The complexity of the SVM model is higher than that of the ANN, but the accuracy of the calculation results can be greatly improved. The theoretical basis of SVM is the relationship of non-linear mapping. A strategy based on structural risk minimization (SRM) can prevent overfitting. For linear indivisible binary classification problems, the optimal algorithm results are obtained by constructing a kernel function. For example, in the schematic diagram of Figure 3, the discriminant function for the two-classification problem is

$$
g(x)=w^{T} x+b
$$

The optimal interface is:

$$
\mathrm{w}^{* \mathrm{~T}} \mathrm{x}+\mathrm{b}^{*}=0
$$

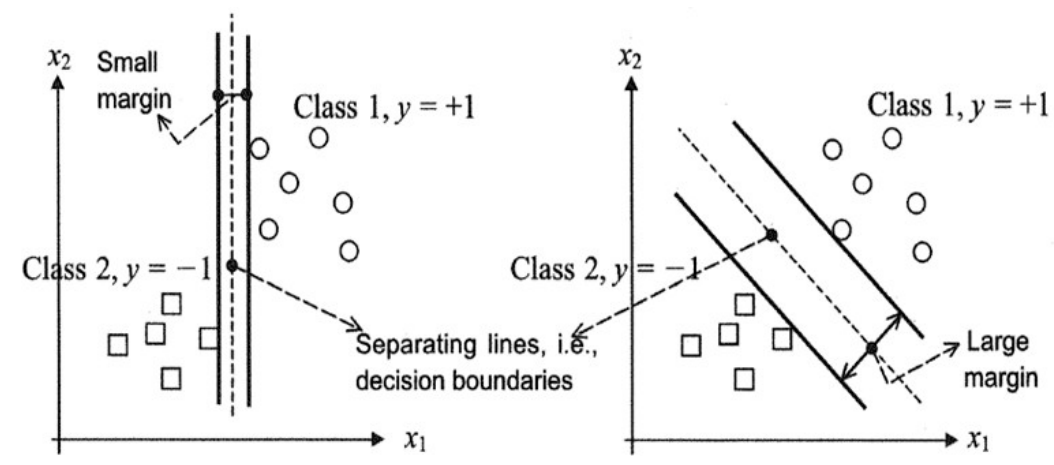

Figure 3. SVM Network Schematic [13]. 


\subsubsection{Machine Learning (ML) Algorithms}

Predicting occupants' thermal comfort by ML algorithms is another key methodology related to artificial intelligence algorithms. Cosma and Simha introduced a non-invasive approach for automatic prediction of personal thermal comfort for real-time feedback with ML [14]. Chaudhuri et al. proposed an indoor-climate control framework to decrease the disparity between energy-efficiency and indoor thermal-comfort in buildings, which comprises two main components: a thermal-comfort prediction model, and an optimization algorithm termed as the optimal air temperature (OAT) algorithm [15]. Lu et al. built a data-driven simulation model for thermal comfort based on k-nearest neighbor (KNN), random forest (RF) and SVM, which they simulate set-point control system with [16]. Gao and Wen introduced a deep learning-based framework for energy optimization and thermal comfort control in smart buildings by considering the energy consumption of HVAC and the thermal comfort of the occupants [17]. Wu et al. investigated an intelligent ensemble machine learning (EML) method Bagging for thermal perception prediction. They compared Bagging approach with ANN and SVM regarding conventional statistical indicators [18]. Kim et al. proposed a personal comfort model to predict individuals' thermal preference by using a new type of feedback, occupants' heating and cooling behaviour with a personal comfort system (PCS) to improve occupant satisfaction and energy use in buildings [19]. Li et al. suggested a methodology by using a camera network to assess thermal comfort in multi-occupancy spaces with defining characteristics of the non-intrusive thermal comfort detection [20]. Megri and Naqa presented a new prediction method for thermal comfort indices by using an improved SVM with more adequate nonlinear kernels to obtain predicted values with a polynomial kernel [21]. Peng and Hsieh developed a platform for simulating occupants' thermal sensations and applied it to examine the performance of SVM on thermal comfort prediction. They also proposed a hybrid SVM-LDA thermal comfort classifier to improve the efficiency of model training [22]. Zhang et al. applied ML to control indoor thermal comfort directly with high accuracy to achieve smart building control and operation [23]. Farhan et al. presented a decision support system to predict human thermal comfort in real-time then suggest corresponding actions. They also introduced a novel ML-based approach to for individual's thermal comfort model [24]. Salamone et al. used multiple methods to assess occupants' thermal comfort through the collection of feedback. Their experiment was the indoor environment in steady-state conditions (PMV and PPD) with the use of nearly and wearable solutions. The method was applied by classification and regression tree (CART) algorithm for machine learning with testing in real offices involving eight workers [25]. Luo et al. summarized the recent literature during the period of 2016-2019, from perspectives of predicted comfort indices, algorithms applied, input features, data sources, sample size, training proportion, predicting accuracy, etc. [26].

\subsection{Feasibility Analysis Applied To Actual Projects}

With the continuous expansion of the building scale and the improvement of the new generation of information technology, more and more intelligent tools are used in the monitoring, control, and analysis of building energy consumption. People's requirements for the indoor living thermal environment are also getting higher and higher, so it is very important to adopt efficient energy consumption design schemes and reduce energy loss. In the process of energy analysis, more rigorous methods need to be adopted. In recent years, the use of building full energy consumption simulation methods to analyze and evaluate various indicators has become a mainstream trend. Creating a comfortable, energy-saving, and healthy living environment has been the focus of people's attention. Building a full energy consumption simulation is different from traditional methods. The new calculation method is based on a dynamic environment and relatively real-time data, and the accuracy of calculation results is greatly improved. But, at the same time, the data required for building full energy simulation is relatively higher. In addition to the typical meteorological year data, hourly data and more detailed thermal performance data are also needed. At present, the popular building full energy simulation software contains EnergyPlus, BLAST, DOE-2 by the United States, Esp-r by Europe, and DeST by 
Tsinghua University in China. Each software has its own advantages and disadvantages, and the diversity of functions helps meet different needs in the energy analysis process. In addition, the full energy consumption simulation strives to have original functions in "integration". Under the current needs of architects and engineers, energy consumption analysis is not only purely mathematical analysis of numbers but also combined with other aspects of the building unit. And, the methodology must make judgments based on complexity thinking. The comfort of the indoor thermal environment is usually measured by Predicted Mean Vote (PMV) index. Previous scholars have conducted in-depth research on PMV. MacArthur et al. firstly proposed a kind of control system and strategy with PMV as the core [27]. Then Schheatzle et al. proposed an optimization-based theory [28]. Simonds et al. proposed the concept of fuzzy control of indoor thermal environment [29] and indoor thermal comfort control based on PMV index.

\subsection{Disadvantages of the Current Research}

Since fuzzy control theory does not need to establish an accurate mathematical model of the target system. Therefore, it is often used in control systems with coupling and nonlinearity. Although the research on the application of fuzzy control in air-conditioning control systems is becoming more and more wide, since the logical reasoning process of fuzzy control is offline, it is difficult to achieve real-time tracking control for the thermal comfort control of an indoor environment. On the other hand, the real-time measurement of temperature, humidity and wind speed is poor, and the remote automatic control cannot be realized.

This study can effectively solve the problems of fuzzy control theory and complete the remote automatic control through real-time "online optimization". As we all know, the comfort of the indoor thermal environment is affected by many factors such as indoor temperature, humidity, wind speed and various outdoor environments. Each parameter has a mutual coupling and non-linear relationship, which constitutes a complex monitoring and control system. Therefore, it is of great significance for room occupants to achieve accurate control of comfort. For heating, cooling, ventilation and lighting of buildings, suitable energy is needed, for this while the reasonable costs must be ensured. Through the method proposed in this paper, it can minimize the consumption of energy generated by heating, cooling, ventilation, and lighting of a room or an area in a building under a predetermined comfort requirement.

\section{Material and Methods}

\subsection{Theory}

This chapter introduces a building intelligent thermal comfort control system based on the Internet of Things. The system contains sensors, controllers, and other IoT devices, and data is collected and analyzed by intelligent algorithms. By using BP neural network algorithm and fitting, the relationship between the actual comfort temperature of the occupants and input/output variables such as PMV is established. Then, the system optimizes and controls the indoor thermal environment. Figure 4 below outlines the process of the system.

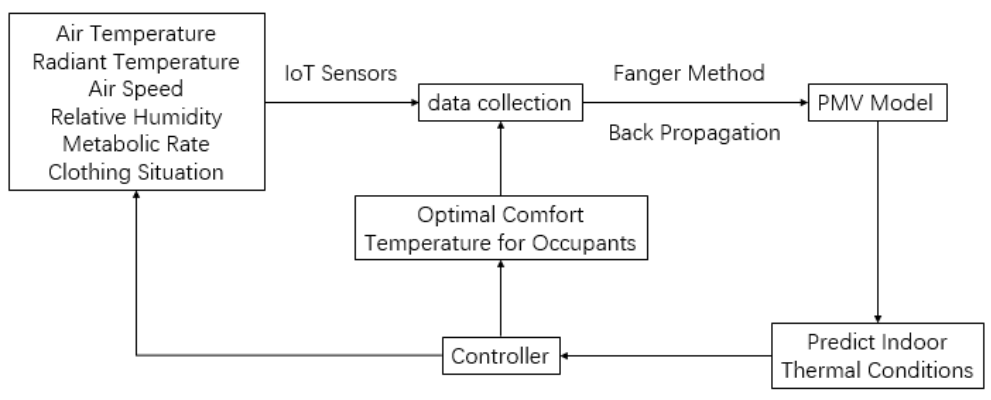

Figure 4. System Working Mode Schematic Diagram. 
Step 1: First use the temperature and humidity detection system to collect data over a period of time, including air temperature (Ta), average radiant temperature (Tr), air velocity (Va), relative humidity (HR), metabolic rate (met), and Clothing situation (clo);

Step 2: Use the data collected in step 1 to obtain PMV values through the Fanger method, and use BP neural network to establish a multivariate logical relationship;

Step 3: Use statistics and analysis methods to establish the relationship between the optimal comfort temperature of the occupants and the PMV value during that period;

Step 4: Predict the trend of the temperature, humidity, wind speed, and PMV value in the room over the next period, and adjust the parameters of each controller to complete the goal of the indoor thermal comfort;

Step 5: Predict the optimal comfort temperature for room occupants over the next period. And compare with the real measurement value.

Step 6: Collect feedback from users to see if they reach the optimal comfort temperature. Finally, it achieves intelligent control of building thermal comfort system.

\subsection{Hardware Devices and Data Collection}

The monitoring system of the method includes a sensor module consisting of a temperature sensor, a humidity sensor, and a wind speed sensor. The module is placed in the area to be measured indoors, and is used to collect air temperature, relative air humidity, and air velocity at the measurement points. The module of the temperature sensor also contains the wet bulb globe thermometer (WBGT) for radiant temperature. 'JTR-10-C WBGT' is used for monitoring the globe radiant temperature and indoor air temperature at the same time. The collected indoor air temperature can be used for mutual calibration with the figure monitored by temperature thermometer. In theory, there are also formulas to convert wet bulb globe thermometer and air temperature for the figure of average radiant temperature (Tr). Here, they don't need to be manually calculated under the convenience of this device. After collecting, the data is sent to a receiving and storage module via a wireless transmitter, and then stored on the computer used. Among them, each module is connected to the power supply equipment to ensure its normal and stable use. The air temperature and humidity sensor use an integrated chip, which can directly output temperature and humidity digital signals. The measurement temperature range is -20 to $60^{\circ} \mathrm{C}$ with the accuracy $\pm 0.5^{\circ} \mathrm{C}$, and the humidity measurement range is 0 to $100 \%$. The wind speed sensor uses a pulsed wind cup anemometer, and the measurement wind speed range is 0 to $50 \mathrm{~m} / \mathrm{s}$. The sensor can collect real-time data in the room and then store it in the analysis system. The parameters of the collected data are classified as follows: air temperature (Ta), average radiant temperature (Tr), air speed (Va), relative humidity (HR), metabolic rate (met), and clothing condition (clo). Figure 5 shows the workflow of data collection and general procedure.

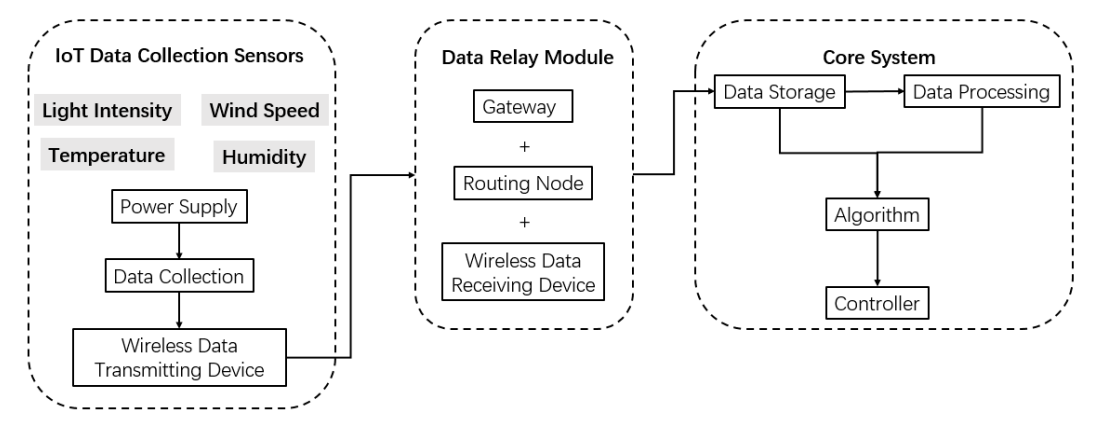

Figure 5. Data Collection and Procedure.

Many of the test instruments and sensors used have standardized data transmission protocols, which are common hardware sensors for the Internet of Things. It is more accurate and scientific from data acquisition to storage. The hardware includes induction acquisition module, light intensity 
sensor, wind speed sensor, temperature sensor, humidity sensor. The control module includes an input terminal, an output terminal, and an interface and a power supply module for connecting various IoT devices. During the measurement and data collection phase, after the multi-parameter sensor group is deployed indoors, the devices and sensors are activated. Then, the measurement command is sent. The measurement command is transmitted to the multi-parameter sensor group through the gateway and the routing node in turn and stored to the computer hardware. The collected data is stored in a CSV (comma-separated values) format by an automated program to facilitate later data processing. In the control phase, after receiving the data, the gateway sends the data to the control module, and the controller operates according to the command and returns the control result.

The experiment started on September 1, 2019 and ended on September 30, 2019, for a total of 30 days. The system starts daily from 8:00 AM to 6:00 PM. Parameters are recorded every 10 minutes, including air temperature (Ta), average radiant temperature (Tr), air speed (Va), relative humidity (HR), metabolic rate (met), and clothing condition (clo). The collected data is divided into two groups: training group and validation group. The experimental site was located in a standard-sized room in Qingdao, China. All IoT sensors used comply with industrial design standards.

The device has a simple structure, low production cost, and wide application range. At the same time, the system runs stably, which can ensure the accuracy of the collected data and real-time transmission and storage.

\subsection{Collection of Optimal Comfort Temperature}

The optimal comfort temperature by indoor occupants is obtained by human-computer interaction system. The system is developed by PHP + MySQL, and the display form is web browser. Figure 6 shows the human-machine interface for collecting indoor optimal comfort temperature. The system is running on a testing computer in the occupant's room. In the test phase, the temperature, humidity, and wind speed of the space where the indoor occupant is located have been obtained by other IoT sensors. This system is mainly used to monitor the real satisfaction of the occupants for the comfort temperature and reflect the real thermal sensation. If the real thermal sensation is not satisfied by the room occupants, the temperature parameters need to be corrected automatically. The system consists of a display interface and buttons. The display interface shows the current room temperature for occupants and test managers to view. Multiple buttons correspond to the following different heat levels.

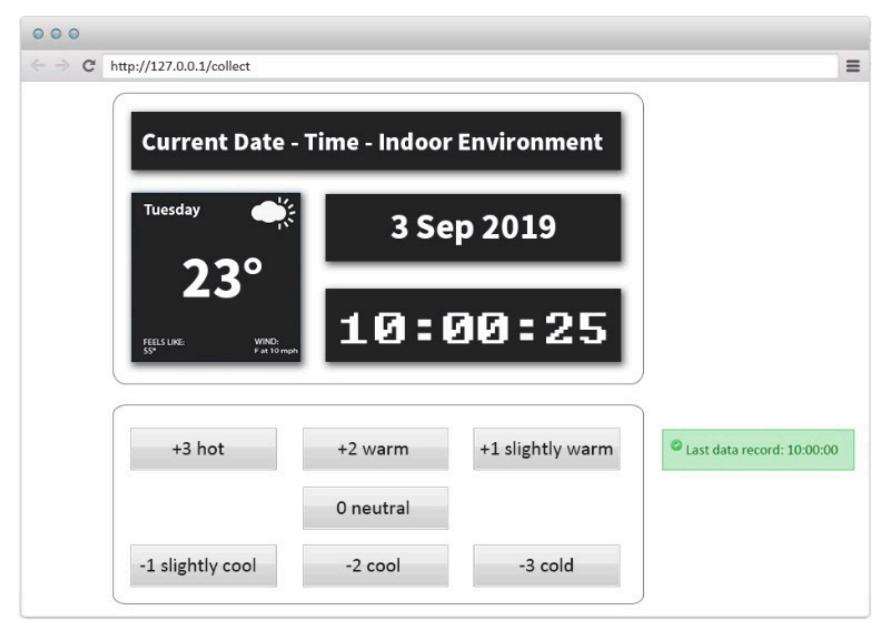

Figure 6. Human-machine Interface for Collecting Indoor Optimal Comfort Temperature.

The interface of the human-computer interaction system is set with 7 different thermal feeling levels, including three "hot" levels, three "cold" levels, and a normal level. These levels are used to reflect the occupants' current feelings about room temperature. The occupants can indicate if they feel 
warm, hot, or too hot, or that they have a cool, cold, or too cold feeling by clicking the corresponding buttons. As input data, clicked levels will be converted into numbers and recorded. It's recorded every 10 minutes, and the average value of the most comfortable temperature for the occupant's click during this period is taken. After receiving the recorded button operation, the controller will reversely control the indoor heating device or cooling device. For example, when the room temperature is 23 degrees, the occupants feel a "cold" feeling. So he clicked the "cold" button. When the system receives the instruction, it will activate the heating device to slowly increase the room temperature. When it reaches 24 degrees, the occupants feel a "cool" feeling. So he clicked the "cool" button. The controller continues to slowly heat to 25 degrees. At this time, if the user feels the best comfortable temperature, click "normal". The system will record the average number of 24.0 degrees as the optimal comfort temperature during these 10 minutes. No action is taken when the occupant is satisfied with his current temperature. The measurement parameters are collected by setting a multi-parameter sensor group, and a curve similarity algorithm is used to calculate the similarity. For curves with high similarity, feature fitting is performed to generate corresponding results.

\subsection{Calculation of Comfort and PMV Value}

Human comfort is related to physiological functions such as sensory function and temperature regulation. At the same time, it is affected by external meteorological factors, such as the temperature, humidity, and pressure of the space in which it is located, the light of the external environment, and the wind speed. When the temperature is moderate, the effects of various parameters on the human body are not significant. But, when the temperature is high or low, its fluctuations become very important to the human body's thermal balance and warmth. Generally speaking, the human body is most sensitive to temperature factors. Therefore, using temperature as a parameter to calculate real-time human comfort can be used as a reference and control for the overall experiment.

The intelligent thermal comfort control system based on the Internet of Things in this paper can be used to control at least one room comfort parameter in a building. During the experiment, a room with a standard size was selected to arrange the sensors and controllers (see Figure 7). This method is suitable for automatic heating, cooling, ventilation, and lighting in the area of a building. A set of modules contain sensors and controllers, which are placed in the area to be measured indoors. The typical room shown in Figure 7 has hardware including induction acquisition module, light intensity sensor, wind speed sensor, temperature sensor, humidity sensor. They are all connected with the central server that can collect data instantly for an indoor environment. The data is sent and received through the wireless gateway, which has already had existed solutions by technological companies. The article is not going to discuss more the data transferring procedure and its format since the main research methodology is applied to thermal comfort considerations. However, in practical applications, multiple sets of control system equipment can be arranged in multiple rooms, so that each room can run an independent thermal comfort control system.

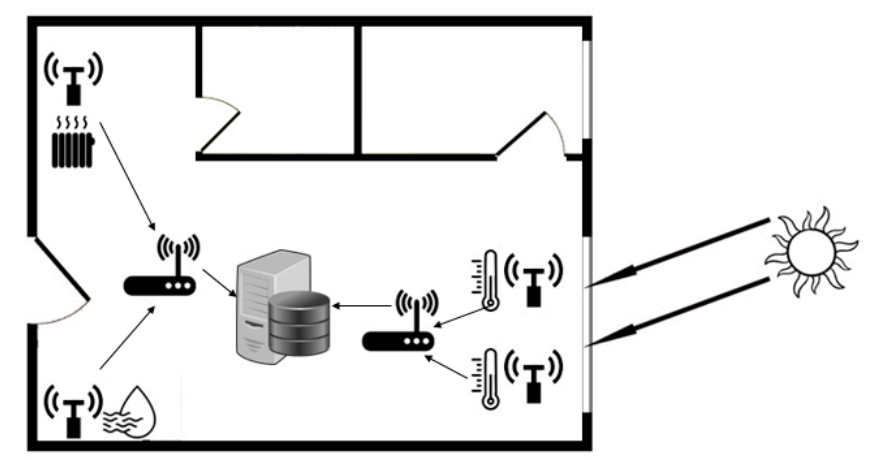

Figure 7. Schematic of A Room with Integrated Automation Devices. 
In order to build a more accurate algorithm, two parameters specific to the occupants of the building are considered, Which is the metabolic rate (met) $\left(1 \mathrm{met}=58 \mathrm{~W} / \mathrm{m}^{2}\right)$ of heat produced by the human body, and the cloning (clo) $\left(1 \mathrm{clo}=0.155 \mathrm{~K} \cdot \mathrm{m}^{2} / \mathrm{W}\right)$ that represents the thermal insulation of clothing. In terms of thermal comfort evaluation, Fanger proposed a method for evaluating thermal sensation models, called PMV (Predicted Mean Vote). The PMV index comprehensively considers various factors that affect the thermal comfort of the human body, and represents the evaluation of thermal comfort by most people. The PMV indicator contains functions of four environmental parameters and two human parameters. Four environmental parameters are internal air temperature (Ta), air velocity (Va), average radiant temperature (Tr), and relative humidity (HR). The two human parameters are metabolic rate (met) and clothing condition (clo). The method proposed by Fanger comprehensively considers the occupants' feelings and environmental parameters, and objectively reflects the thermal phenomenon of the surrounding environment (Figure 8).

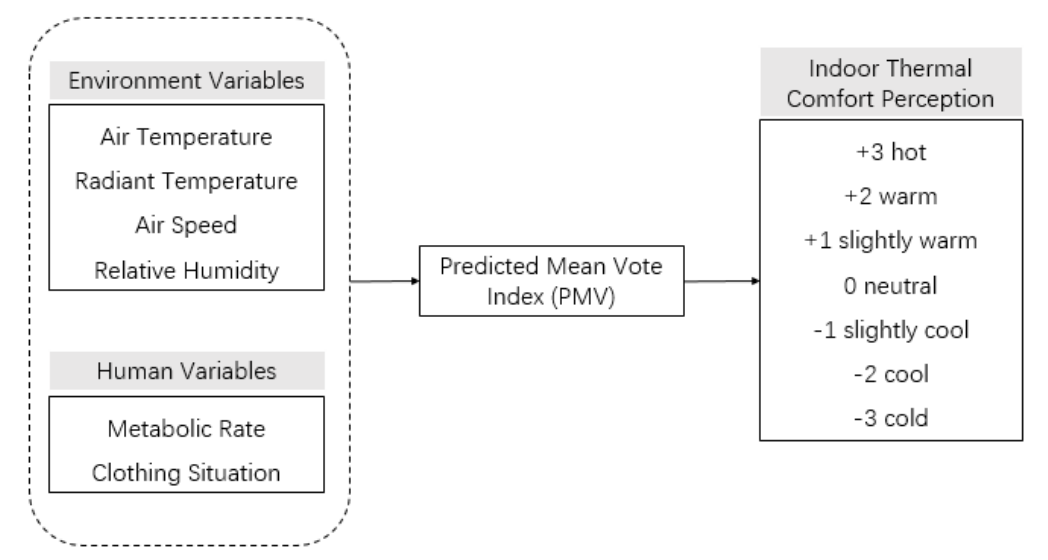

Figure 8. Relationship Between Thermal Comfort and PMV Value.

In the Python programming language environment, the formula for calculating PMV is as follows: $\mathrm{a}=0.028+0.3033 * \mathrm{np} \cdot \exp \left(-0.036^{*} \mathrm{~m}\right)$

$\mathrm{b}=0.028+0.3033 * \mathrm{np} \cdot \exp (-0.036 * \mathrm{~m})$

$\mathrm{c}=0.42 *(\mathrm{~m}-\mathrm{w}-58.15)-0.0000173 * \mathrm{~m} *(5867-\mathrm{pa})-0.0014 * \mathrm{~m} *(34-\mathrm{ta})$

$\mathrm{d}=3.96 * 10 *(-8) * \mathrm{fcl}^{*}((\mathrm{tcl}+273) * * 4-(\mathrm{tmrt}+273) * * 4)-\mathrm{fcl}^{*} 12.1 * \mathrm{va} * 0.5 *(\mathrm{tcl}-\mathrm{ta})$

$\mathrm{pmv}=\mathrm{a} *(\mathrm{~b}-\mathrm{c}-\mathrm{d})$;

\subsection{Building BP (Back Propagation) Neural Networks}

First, normalize the obtained data to the output. $x_{\min }$ is the minimum value of $\mathrm{x}$, and $x_{\max }$ is the maximum value of $x$. Use the mapminmax function to normalize, and the final output is also normalized data. Map the results on [0,1]. The normalization formula is as follows

$$
y=\frac{x-x_{\min }}{x_{\max }-x_{\min }}
$$

The Python code for this function is

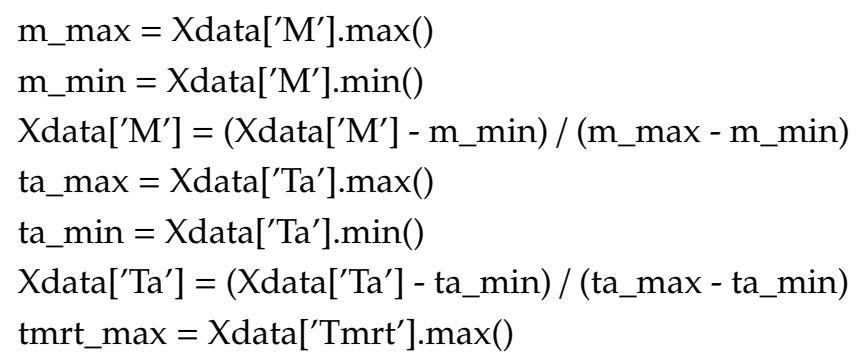




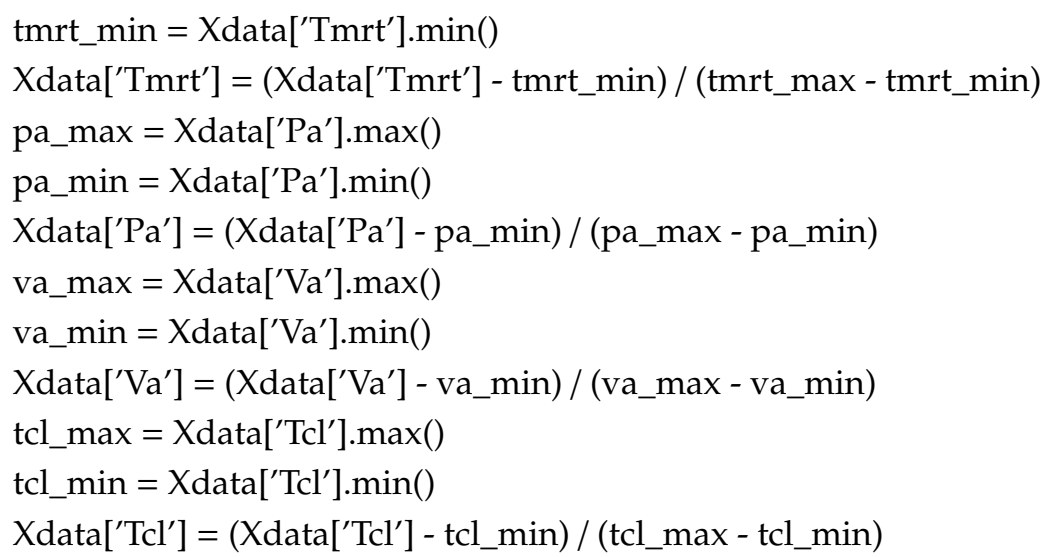

This method is based on Python and uses a BP neural network to build a PMV prediction model. The sample data is divided into training group and verification group, with a total of 1800 group of dataset. The first 1700 groups are selected as the training sample set to learn and train the prediction model. In addition, 100 sets of data were selected as the test sample set for verifying the accuracy of the prediction model. Select the air temperature $(\mathrm{Ta})$, average radiant temperature $(\mathrm{Tr})$, air velocity (Va), relative humidity (HR), metabolic rate (met), and clothing (clo) in the sample as input variables of the prediction model. The pmv prediction is used as the model's output variable y. Which means, the established three-layer BP neural network energy prediction model has six input vectors and one output vector. The code is as follows:

X_train_valid $=$ Xdata[0: 1700]

PMV_all = Xdata['PMV'].to_numpy()\# Splitting into training and validation data

X_train, X_valid, y_train, y_valid = train_test_split(X_train_valid.drop (['Record no', 'PMV', 'Ttest'], axis $=1)$, X_train_valid['PMV'], test_size $=0.20$, random_state $=101)$

The network training parameters are set with the maximum number of iterations to 2500 and the training target is set to 6 . According to the formula

$$
n_{1}=\mathrm{n}+\mathrm{m}+\mathrm{a}
$$

$n_{1}$ is the number of hidden layers, $\mathrm{n}$ is the number of input layers, $\mathrm{m}$ is the number of output layers, $a$ is a constant between [1,10], that is, the number of hidden layers The maximum is 10 . When the number of hidden layers is 1 , the network does not converge and can be ignored. Therefore, we only need to compare the sum of squared errors when the number of hidden layers is $[2,10]$. The training process is as follows:

nnmodel $=$ MLPRegressor(solver $=$ 'adam', learning_rate_init $=0.001$, alpha $=0.0$, hidden_layer_sizes $=(10,10,10)$, random_state $=1$, max_iter $=2500$, tol $=1 \mathrm{e}-6)$

nnmodel.fit(X_train, y_train)

y_train_predict $=$ nnmodel.predict $\left(X \_\right.$train $)$

The Python libraries used in this experiment are Pandas, Numpy, MLPRegressor, train_test_split, plt, r2_score.

\section{Results}

The collected 1,700 sets of training data start at 8 am on September 1, 2019 and end at 11:00 am on September 29, 2019. They are recorded every 10 minutes for 10 hours a day. Due to space limitations, only the first 10 sets of data are shown in Tables 1 and 2 below: 
Table 1. Indoor Data Collected by Sensors.

\begin{tabular}{cccccccc}
\hline No. & Time & met & Ta & Tr & HR & Va & clo \\
\hline 1 & $2019 / 9 / 18: 00$ & 69.1 & 21.0 & 21.0 & 1328 & 0.11 & 20.0 \\
2 & $2019 / 9 / 18: 10$ & 72.5 & 21.2 & 21.9 & 1341 & 0.11 & 21.0 \\
3 & $2019 / 9 / 18: 20$ & 71.8 & 22.1 & 21.5 & 1368 & 0.12 & 20.6 \\
4 & $2019 / 9 / 18: 30$ & 72.5 & 21.7 & 21.7 & 1341 & 0.11 & 20.4 \\
5 & $2019 / 9 / 18: 40$ & 71.8 & 21.9 & 21.9 & 1341 & 0.11 & 20.4 \\
6 & $2019 / 9 / 18: 50$ & 71.1 & 21.7 & 21.9 & 1394 & 0.12 & 20.2 \\
7 & $2019 / 9 / 19: 00$ & 72.5 & 21.7 & 21.7 & 1394 & 0.11 & 20.6 \\
8 & $2019 / 9 / 19: 10$ & 71.1 & 21.7 & 21.2 & 1381 & 0.11 & 20.6 \\
9 & $2019 / 9 / 19: 20$ & 71.8 & 21.2 & 21.5 & 1394 & 0.12 & 20.8 \\
10 & $2019 / 9 / 19: 30$ & 71.8 & 22.1 & 21.9 & 1355 & 0.12 & 21.0 \\
\hline
\end{tabular}

Table 2. Collection of Optimal Comfort Temperature by Human-computer Interaction System.

\begin{tabular}{cccccc}
\hline No. & Time & Ttest & No. & Time & Ttest \\
\hline 1 & $2019 / 9 / 18: 00$ & 26.0 & 26 & $2019 / 9 / 112: 10$ & 26.3 \\
2 & $2019 / 9 / 18: 10$ & 26.6 & 27 & $2019 / 9 / 112: 20$ & 26.6 \\
3 & $2019 / 9 / 18: 20$ & 27.1 & 28 & $2019 / 9 / 112: 30$ & 26.6 \\
4 & $2019 / 9 / 18: 30$ & 26.8 & 29 & $2019 / 9 / 112: 40$ & 26.8 \\
5 & $2019 / 9 / 18: 40$ & 26.6 & 30 & $2019 / 9 / 112: 50$ & 26.3 \\
6 & $2019 / 9 / 18: 50$ & 27.1 & 31 & $2019 / 9 / 113: 00$ & 25.1 \\
7 & $2019 / 9 / 19: 00$ & 27.3 & 32 & $2019 / 9 / 113: 10$ & 25.3 \\
8 & $2019 / 9 / 19: 10$ & 27.1 & 33 & $2019 / 9 / 113: 20$ & 25.8 \\
9 & $2019 / 9 / 19: 20$ & 27.3 & 34 & $2019 / 9 / 113: 30$ & 25.6 \\
10 & $2019 / 9 / 19: 30$ & 26.8 & 35 & $2019 / 9 / 113: 40$ & 26.3 \\
\hline
\end{tabular}

After training the data using the above model, the results are as follows Figure 9 above shows the results of the variation trend of the error convergence caused by model training. It can be seen from the error curve in the figure that in the initial training period, the error decreases very quickly, but as the cumulative error decreases to a certain extent, the further decline will be very slow. The simulation results show that when the iteration is performed up to 1100 times, the sum of squared errors is the smallest. The error reaches a stable value and ends. According to the theory of machine learning algorithms, when the accuracy requirements are high, using cumulative errors to change the weights and thresholds will increase the number of training. When the samples are small or the accuracy is not high, the cumulative error for weight update can greatly speed up the training.

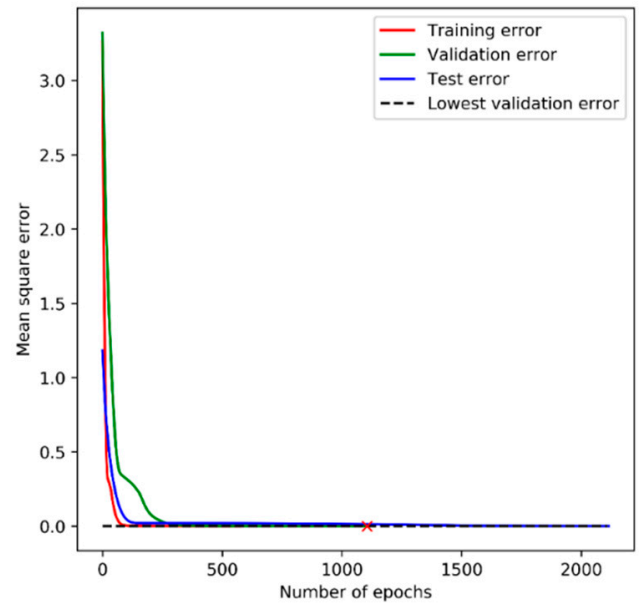

Figure 9. Error Convergence Curve. 
Figure 10 fits the training data set, the test data, and the test data set. Because the time selected for data collection is relatively concentrated, the span is not large. Therefore, it can be seen that the objective function has a highly linear relationship with the predicted value. It can be seen from Figure 11 that in 100 sets of test samples, the calculated data of the PMV equation is very close to the data predicted by the neural network. Comparing the experimental results, it can be seen that the prediction accuracy of PMV is high, and it has a good nonlinear fitting ability. It objectively reflects the relationship between PMV and various parameters of the indoor environment and proves that it is feasible to introduce BP network into PMV prediction research. It can be applied to the next stage of prediction experiments.
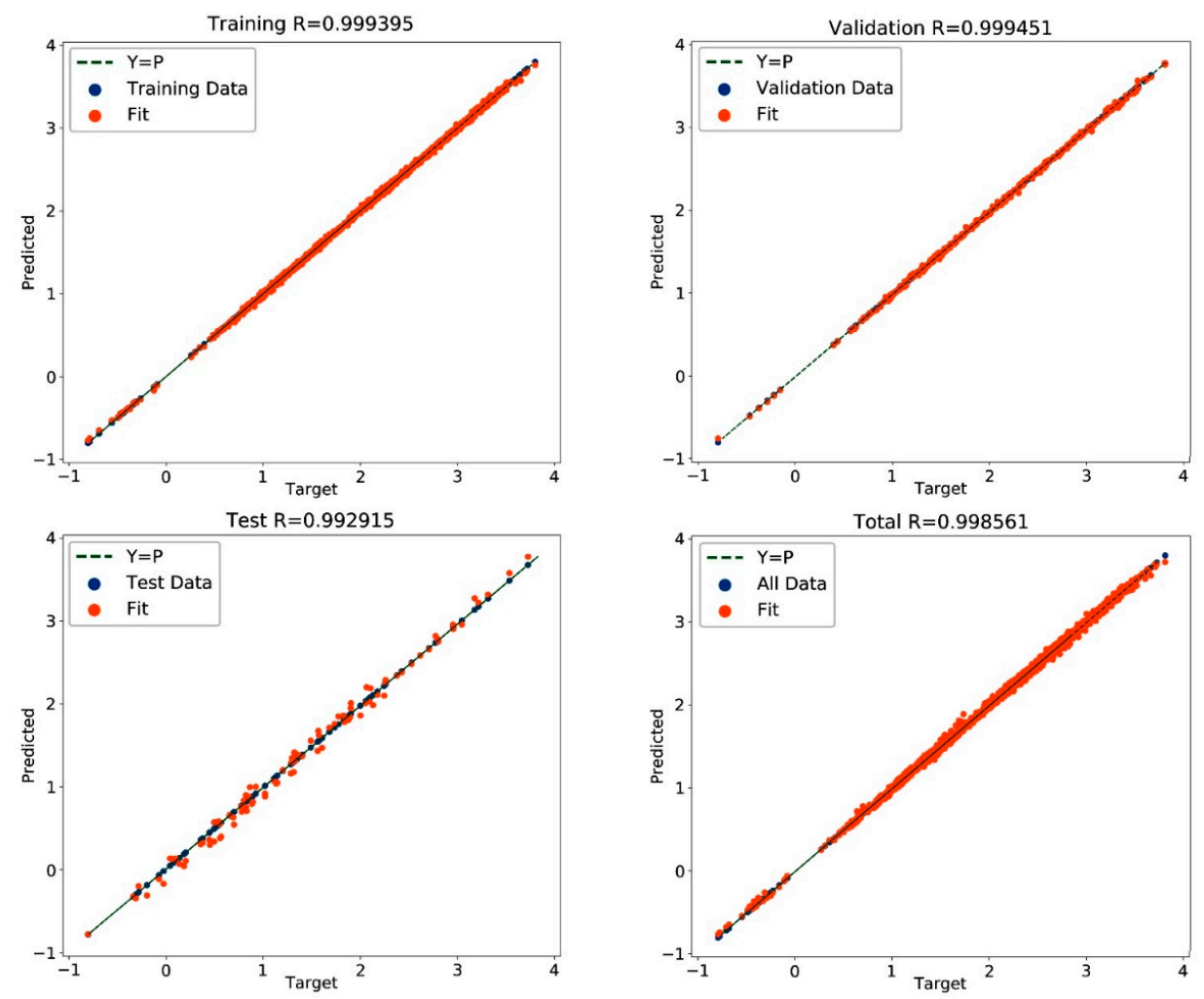

Figure 10. Error Histogram.

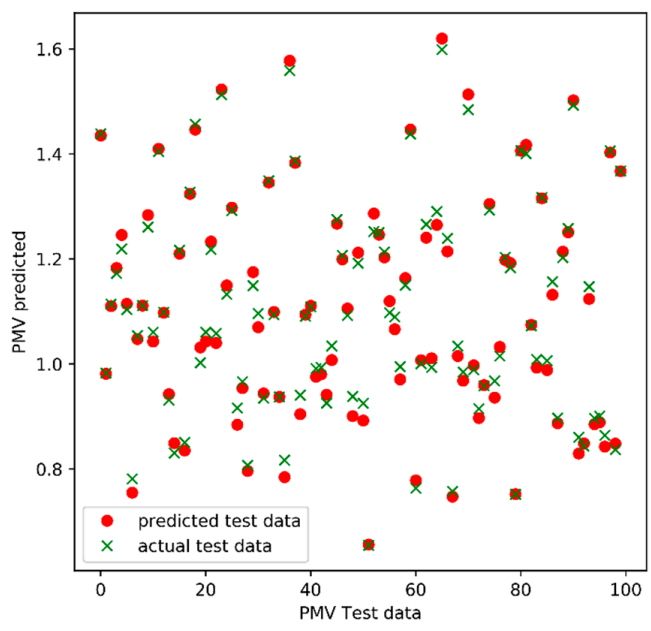

Figure 11. Comparison of Actual Output and Expected Value of PMV. 


\section{Discussion}

The hardware equipment is highly integrated, practical, low cost, and flexible to improve the comfort of occupants. The advantage of this system is that it combines multiple test instruments and sensors to measure parameters related to the indoor environment. And can help the occupants automatically control the indoor temperature to achieve the best comfortable temperature. Compared with a single thermal environment comfort evaluation, the system is more accurate and scientific, with higher accuracy. By establishing the parameters of mutual coupling and a non-linear relationship, multivariable operation is performed based on BP and PMV. The hardware devices are in low production cost but with a wide range of use.

The technology based on the Internet of Things and artificial intelligence algorithms has greatly accelerated the development of the building industry. IoT is an abbreviation of Internet of Things, which means that traditional buildings are connected with intelligent hardware, with data as a carrier, and a variety of analysis software. To informatize the operation of the building, sensors like NFC, RFID, etc are commonly used. The Internet of Things technology can help owners and operators to efficiently manage building spaces and places of use, effectively improve the work efficiency of relevant personnel, and solve pain points in the industry. The Internet of Things technology mainly uses sensors such as RFID and NFC to control the building process. Buildings combined with the Internet of Things can increase their data use efficiency and monitor the use of various items in real time. Advanced building networking systems are often closely linked to Building Management Systems (BMS), bringing great convenience to owners and operators of property rights. It can also improve the efficiency of the use of hardware and the degree of informationization of the Internet of Things hardware. For example, real-time monitoring and adjustment of temperature in the building, heating and cooling conditions, monitoring and management of visitors. Each intelligent component in a building represents an independent system, such as access control, security. Without the intelligent connection of intelligent buildings at the software level, too many independent operating systems will seriously delay the efficiency of hardware use, while consuming more energy.

Building automation cannot be separated from the Internet of Things. The traditional building design process is followed by the construction process. The Internet of Things helps to better manage and monitor data and reduce energy consumption. With the continuous iterative improvement of technology, the architectural design industry is increasingly relying on intelligent buildings for its efficient and powerful data ecosystem. Intelligent programs can feedback the running status of the building in real-time to meet the needs of building occupants. Therefore, there is a huge business opportunity in the intelligent building market. Intelligent buildings that allow users to customize functions may become the mainstream benchmark in the industry in the future. Due to the poor timeliness and occlusion of traditional information transmission, real-time data transmission cannot be achieved. Once problems occur, they cannot be learned and resolved in time, but after the application of the Internet of Things technology, such problems can be quickly resolved, and major social problems are avoided. The use of Internet of Things technology can also predict potential problems in the later stages of construction and prevent them in advance. Since the construction design drawing is directly related to the quality of the project, the combination with BIM technology can be used for the automated data review of the construction drawing [30].

Artificial intelligence algorithms are a means to solve problems in batches. Usually, a feasible algorithm includes input, algorithm part, output. Artificial intelligence algorithms originated from the interdisciplinary subject of computer and mathematics. Before the rise of computer hardware development, they have appeared in the field of mathematical research. Usually, an algorithm is to build a mathematical model, which can concretely abstract the problem and solve it rigorously using mathematical logic. In the field of mathematics, from the initial SAS, SPSS to various macro operations, to the current use of various computers into languages, such as Python, R Language. So, artificial intelligence is not limited to refer to the same platform and the same programming language. For artificial intelligence, data is a very important resource, because algorithms are based on data to obtain 
results. Only enough data can diversify results and minimize deviations. Computer "algorithms" are based on mathematical "algorithms". It was originally developed with the idea of solving problems with mathematical models, so the practical application of artificial intelligence cannot be separated from the research and development of mathematical models. The programming language used for artificial intelligence is not single, there may be multiple solutions to the same problem, and there is not only one solution. The correct process is essential to achieve reasonable results. For computers with the same configuration, those with high processing efficiency can get the calculation results first, which can greatly shorten the feedback cycle of the calculation.

The mature underlying framework and open source code can enhance the innovation ability of the artificial intelligence industry, so the core of artificial intelligence is algorithms. Regardless of the strength of the hardware or the amount of data, only a suitable algorithm can make the best use of the hardware. Artificial intelligence algorithms are a series of operational processes that are performed rigorously and step by step by the computer. The mathematical model, algorithm design, and training are used to reason and calculate, thereby solving complex problems. Machine learning is an application of artificial intelligence. Algorithms can automatically learn related data to find fixed rules and then solve the problem of logical relationships behind the data set. Machine learning usually use methods such as decision trees, naive Bayes classification, and least squares regression. So, finding the right algorithm is very important, it's like a craftsman's craft. Algorithms are used to reasonably clean and classify the data, so as to select the data that meets the requirements for learning.

Whether it is the Internet of Things or artificial intelligence algorithms, in the process of information development, the most important thing is data. Traditional data processing has been criticized for its complexity and complexity. The proposal of big data technology has effectively solved the problems of structured and unstructured data processing, and improved the technical means of acquisition, sorting and processing to a more strategic and forward-looking position. According to the different characteristics of different industries, big data can also be transformed into its own advantages, starting from three aspects, scale, speed, and efficiency. Big data is dedicated to extracting and using valuable information [31], providing important solutions for enterprises or scientific research institutions. Big data upgrades the traditional "database", which is the main research area of the current technology industry. The big data industry has become one of the biggest opportunities at present, and new technologies bring opportunities for transformation in various industries, fully exploring their potential, and accelerating the importance of data in the industry. Big data is characterized by its large scale and large volume. At present, mainstream data is calculated at the level of trillions and billions. This cutting-edge discipline that leads the technology trend is also widely used in cross-cutting research.

Big data mainly refers to the classification of structured or unstructured large and diverse data sets, which is very different from traditional data processing methods. At present, big data has been integrated into all parts of life, and it is a technology trend that is quite concerned in the field of building. The algorithm-based practice provides technical support for the management and centralized processing of large quantities of data, so it is widely used in computer technology and interdisciplinary subjects. Whether it is the policy support of the local government or the developing strategy of enterprises in the industry, big data will undoubtedly promote the accelerated development of the industry. However, those opportunities also bring many challenges to the iterative advancement of technology. Many high-growth big data companies have entered the adjustment period, and even some companies' big data businesses are facing compliance investigations, so higher-priority regulatory policies need to be issued accordingly.

Intelligent Thermal Comfort Controlling System is the combined application direction of the interdisciplinary of big data, artificial intelligence and Internet of Things mentioned above. Intelligent buildings using IoT modules can effectively control the thermal comfort of indoor spaces and improve energy efficiency. HVAC and lighting are the main construction and operation costs. With the promotion of new technologies, it can intelligently solve the process from design to construction, integrate decentralized projects on the same platform, and greatly improve work efficiency. New 
technologies have brought innovation to traditional industries. At the same time, architectural designers are also facing the challenges and foundations of new technologies. Data-based intelligent design algorithms have gradually moved from the field of computer science to the field of building practice. For example, a deep learning library can analyze the style of architectural photos to intelligently generate architectural designs and construction drawings, and gradually improve the later construction process. For architects, the use of big data technology across disciplines can achieve rapid communicating and improve work efficiency. Big data can realize multi-scenario data analysis and data sharing through multiple nodes and frameworks, and solve problems encountered during design. The combination with intelligent hardware can also control the temperature and personalized lighting in the building, so that the data in the building can be connected and used efficiently, reducing the operating cost of the property owner and increasing the economic value. The importance of data adds much convenience to the field of smart buildings, which provides more potential opportunities for the building industry.

\section{Conclusions and Future Work}

Among the methods for building energy prediction based on artificial intelligence technology, each algorithm has advantages and disadvantages. How to choose a specific intelligent algorithm for different needs becomes important. This paper analyzes different model prediction methods and proposes a combined algorithm for real-time analysis of short-term energy consumption prediction, which reflects the importance of fast prediction. Fast and effective analysis of building energy consumption is achieved through feedback correction of predicted values. It is helpful for the occupants of the building to make adjustments based on the feedback in a timely manner and has certain theoretical guiding significance and practical application value. Although the method in this paper only performs data collection and analysis for a single room, in practical applications, it can be arranged to multiple rooms, multiple floors, and even individual buildings. The method has high scalability. The experimental method covers systems including heating and air conditioning equipment, as well as independent ventilation equipment.

However, this methodology of this research still has some limitations. In the data verification part, some untested samples were selected for verification. From the results, there is a certain error (about $3 \%$ ) between the expected value and the actual value, which may be caused by two reasons.

- The accuracy of the BP algorithm still needs to be improved. This is limited by computer hardware conditions. At present, it is impossible to achieve infinite precision to achieve extreme accuracy.

- In the current case study, it concerns more about the influence of the temperature for thermal comfort, while the influence of air velocity was neglected. The air velocity could become an important variable for assessing thermal comfort. Relatively, the complexity of the monitoring system will be greatly increased.

- The experimental site cannot be strict and accurate. Due to the hardware problems of the sensors and controllers, errors may occur when recording and storing data.

In the future, the research direction of intelligent thermal comfort control systems for buildings based on the Internet of Things will gradually increase with the development of computer hardware. This will make the prediction model more accurate so that the predicted value is closer to the actual value.

Moreover, the current system is researched on thermal comfort considerations by using computational methods. Besides the virtual experiments in a lab environment, however, it's highly demanding to apply with related research with multi-objective simulations, for instance, the facades, construction process. Bedon et al. presented the study on a special system with a sandwich design made of glass fibre-reinforced polymer (GFRP) pultruded profiles. Their novel design concept has up to over $10 \%$ and $15 \%$ respectively improvement on thermal and structural performances compared to traditional non-integrated systems [32]. Combining with actual building projects is the best practice direction of the system. Bedon et al. collected some recent examples and design concepts of adaptive systems with classification for structural assessment and adaptive facades of buildings [33]. No 
matter indoor or exterior system, the most fundamental aim is to predict or control building energy consumption in reasonable and efficient methods. In future research, the proposed framework and system for detecting the indoor environmental variables related to this research will introduce more variables based on the various types of algorithms. More hardware will also be applied to the controllers. Therefore, it can be applied to different rooms or different areas of the same space, as each area has an internal adjustment terminal. Further extended investigations are required to exploit and optimize the potential of the system.

Author Contributions: In this paper, Y.Z., P.V.G. and Z.L. conceived the idea. Y.Z. conceived and designed the experiments; Y.Z. performed the experiments. All the authors (Y.Z., P.V.G. and Z.L.) organized the work; analyzed the experiments. Z.L. reviewed the writing of the paper, its structure. All authors have read and agreed to the published version of the manuscript.

Funding: This research received no external funding.

Conflicts of Interest: The authors declare no conflict of interest.

\section{References}

1. Zhao, Y. Analysis of The Application Of Big Data And Smart City Technology in Urban Planning. Archit. C. 2016, 8, 202-203.

2. Yang, L. Analysis of the Application of Big Data in Intelligent Buildings. China Real Estate Ind. 2018, $18,37$.

3. Hu, C. Research on Data-Driven Prediction Method of Building Energy Consumption; Jiangsu University: Jiangsu, China, 2016.

4. Neto, A.H.; Fiorelli F A, S. Comparison between detailed model simulation and artificial neural network for forecasting building energy consumption. Energy Build. 2008, 40, 2169-2176. [CrossRef]

5. Kalogirou, S.A.; Bojic, M. Artificial neural networks for the prediction of the energy consumption of a passive solar building. Energy 2000, 25, 479-491. [CrossRef]

6. Chen, Y.; Liu, J.; Li, X.; Yin, B.; Wu, X. HVAC System Energy Consumption Prediction of Green Office Building Based on ANN Method. Build. Energy Sav. 2017, 10, 1-5.

7. Yu, W.; Li, B.Z.; Yang, M.Y. Building Multi-Objective Forecasting Model Based on Artificial Neural Network. J. Central South Univ. Nat. Sci. Ed. 2012, 12, 4949-4955.

8. Zhang, X. Neural Network Model with Dynamic Compensation and Its Application in Dynamic System Modeling. Control Theory Appl. 1996, 13, 823-826.

9. Li, S.; Liu, H. Generalized predictive control based on neural network error correction. Control Theory Appl. 1996, 13, 677-680.

10. Ahmad, A.; Hassan, M.; Abdullah, M.; Rahman, H.A.; Hussin, F.; Abdullah, H.; Saidur, R. A review on applications of ANN and SVM for building electrical energy consumption forecasting. Renew. Sustain. Energy Rev. 2014, 33, 102-109. [CrossRef]

11. Dong, B.; Cheng, C.; Lee, S.E. Applying support vector machines to predict building energy consumption in tropical region. Energy Build. 2005, 37, 545-553. [CrossRef]

12. Li, Q.; Meng, Q.; Cai, J.; Yoshino, H.; Mochida, A. Applying support vector machine to predict hourly cooling load in the building. Appl. Energy 2009, 86, 2249-2256. [CrossRef]

13. Comparison of MLP, RBF, SVM Networks and Their Application Prospects. Available online: https://blog.csdn.net/xiaoding133/article/details/9079103 (accessed on 1 December 2019).

14. Cosma, A.; Simba, R. Machine learning method for real-time non-invasive prediction of individual thermal preference in transient conditions. Build. Environ. 2019, 148, 372-383. [CrossRef]

15. Chaudhuri, T.; Soh, Y.; Li, H.; Xie, L. A feedforward neural network based indoor-Climate control framework for thermal comfort and energy saving in buildings. Appl. Energy 2019, 248, 44-53. [CrossRef]

16. Lu, S.; Wang, W.; Lin, C.; Hameen, E. Data-driven simulation of a thermal comfort-based temperature set-point control with ASHRAE RP884. Build. Environ. 2019, 156, 137-146.

17. Gao, G.; Li, J.; Wen, Y. Energy-efficient thermal comfort control in smart buildings via deep reinforcement learning. arXiv 2019, arXiv:1901.04693v1.

18. Wu, Z.; Li, N.; Peng, J.; Cui, H.; Liu, P.; Li, H.; Li, X. Using an ensemble machine learning methodology-Bagging to predict occupants' thermal comfort in buildings. Energy Build. 2018, 173, 117-127. [CrossRef] 
19. Kim, J.; Zhou, Y.; Schiavon, S.; Raftery, P.; Brager, G. Personal comfort models: predicting individuals' thermal preference using occupant heating and cooling behavior and machine learning. Build. Environ. 2018, 129, 96-106. [CrossRef]

20. Li, D.; Menassa, C.; Kamat, V. Robust non-intrusive interpretation of occupant thermal comfort in built environments with low-cost networked thermal cameras. Appl. Energy 2019, 251, 113336. [CrossRef]

21. Megri, A.; Naqa, I. Prediction of the thermal comfort indices using improved support vector machine classifiers and nonlinear kernel functions. Indoor Built Environ. 2014, 25, 6-16. [CrossRef]

22. Peng, B.; Hsieh, S. Data-driven thermal comfort prediction with support vector machine. In Proceedings of the 12th International Manufacturing Science and Engineering Conference, Los Angles, CA, USA, 4-8 June 2017.

23. Zhang, W.; Liu, F.; Fan, R. Improved thermal comfort modeling for smart buildings: a data analytics study. Int. J. Electr. Power Energy Syst. 2018, 103, 634-643. [CrossRef]

24. Farhan, A.; Pattipati, K.; Wang, B.; Luh, P. Predicting individual thermal comfort using machine learning algorithms. In Proceedings of the International Conference on Automation Science and Engineering, Gothenburg, Sweden, 24-28 August 2015.

25. Salamone, F.; Belussi, L.; Currò, C.; Danza, L.; Ghellere, M.; Guazzi, G.; Lenzi, B.; Megale, V.; Meroni, I. Integrated method for personal thermal comfort assessment and optimization through users' feedback, IoT and machine learning: a case study. Sensors 2018, 18, 1602. [CrossRef] [PubMed]

26. Luo, M.; Xie, J.; Yan, Y.; Ke, Z.; Yu, P.; Wang, Z.; Zhang, J. Comparing machine learning algorithms in predicting thermal sensation with ASHRAE Comfort Database II. Energy Build. 2020. [CrossRef]

27. Macarthur, J.W. Humidity and predicted-mean-vote-based comfort control. ASHRAE Trans. 1986, 92, 7-17.

28. Scheatzle, D.G.; Arch, D. The development of PMV-based control for a residence in a hot and arid climate. ASHRAE Trans. 1991, 97, 1002-1019.

29. Simmonds, P. Thermal Comfort and Optimal Energy Use; ASHRAE: Atlanta, GA, USA, 1993; pp. 037-1048.

30. Zhao, Y.; Ji, L.; Lu, W. Research on the quality process of steel structure construction in construction engineering and safety countermeasures. Value Eng. 2011, 30, 92.

31. Zhao, Y.; Chen, Y. Research and Design Thinking on Energy-efficient Buildings Based on Big Data Technology. Build. Energy Effic. 2017, 2, 109-111.

32. Bedon, C.; Agullo, C.P.; Luna-Navarro, A.; Overend, M.; Favoino, F. Thermo-mechanical investigation of novel GFRP-glass sandwich facade components. In Challenging Glass Conference Proceedings; TU Delft Open: Delft, The Netherlands, 2018; pp. 501-512.

33. Bedon, C.; Honfi, D.; Machalická, K.V.; Eliášová, M.; Vokáč, M.; Kozłowski, M.; Wüest, T.; Santos, F.; Portal, N.W. Structural characterisation of adaptive facades in Europe-Part I: Insight on classification rules, performance metrics and design methods. J. Build. Eng. 2019, 25, 100721. [CrossRef] 